\title{
Los efectos del proceso de limpieza en las estructuras pictóricas
}

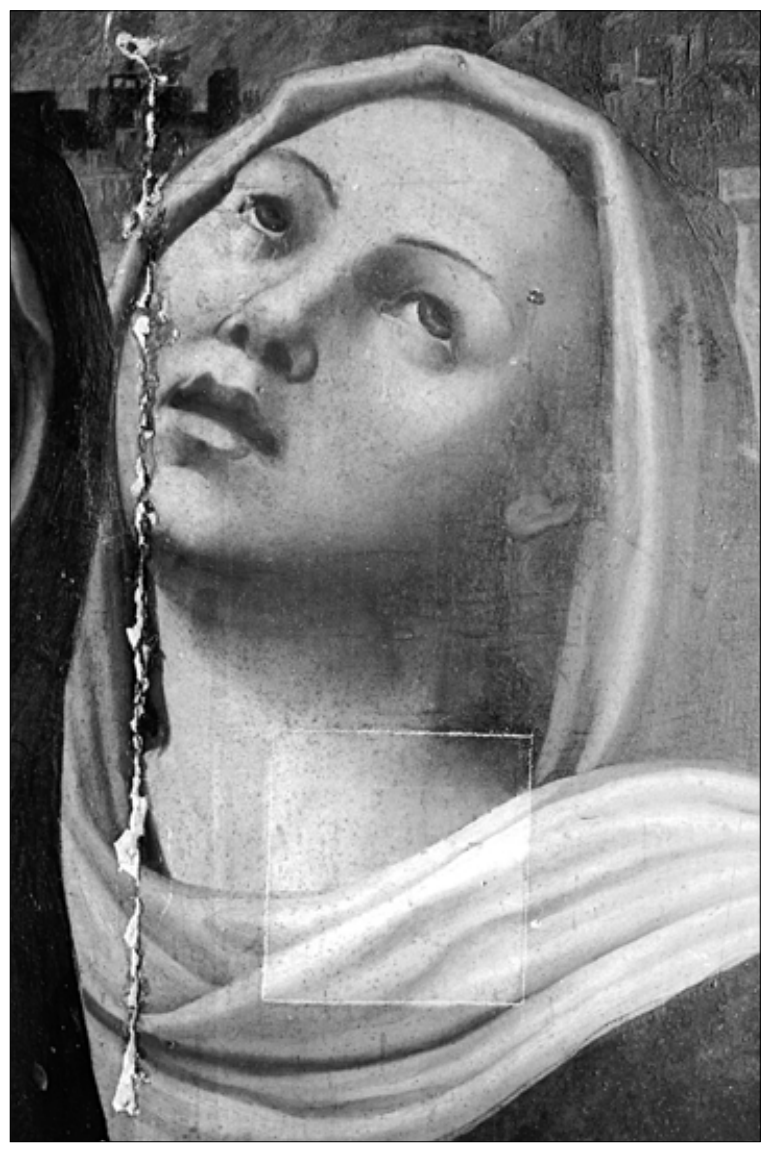

José Manuel Barros García

Dr. en Bellas Artes Restaurador de pintura

\section{Resumen}

En este artículo se analiza la cuestión de los posibles efectos deletéreos del proceso de limpieza en estructuras pictóricas. Se revisan algunas de las principales líneas de investigación acerca de este complejo problema, en especial las centradas en la extracción de compuestos solubles y en la retención de residuos. La lixiviación ha sido estudiada en relación con las pinturas al óleo, aunque también empieza a mostrarse un mayor interés con respecto al estudio del efecto de los disolventes sobre estructuras pictóricas elaboradas con otros aglutinantes. La retención de residuos y sus efectos a largo plazo, es otro de los temas más frecuentes en los últimos años, en especial desde la introducción en la práctica habitual de la restauración de los sistemas diseñados por Richard Wolbers. Los resultados de todas estas investigaciones ponen en un primer plano la necesidad de una metodología rigurosa y unificada, tanto al examinar los posibles efectos de la limpieza como en el propio proceso de restauración.

\section{Palabras clave}

Limpieza / Estructura Pictórica / Disolvente / Lixiviación / Tensoactivo / Gel / Residuo

\section{Introducción}

Una de las cuestiones planteadas, al estudiar los procedimientos de restauración, es la de la inocuidad de 
la operación. El proceso de limpieza consiste en la extracción selectiva de todos o parte de los materiales presentes en la estructura superficial. En este caso, la inocuidad consistiría en no involucrar a los demás materiales $y$, de forma muy especial, a los que componen la estructura pictórica.

Estas cuestiones, referentes a la seguridad de la pintura, han sido muy habituales en todas las controversias acerca del problema de la limpieza desde el s. $X V I I I$, e incluso antes. Hoy en día, todavía es uno de los temas más analizados y discutidos, aunque la dificultad en establecer unas conclusiones claras y la presencia de resultados (aparentemente) contradictorios ha generado un cierto cansancio entre restauradores e investigadores.

Un ejemplo de esos resultados (aparentemente) contradictorios lo tenemos en dos artículos publicados en los últimos años en Studies in Conservation. En 1998, White y Roy publicaron los resultados de un extenso estudio acerca de los efectos de la limpieza en pinturas al temple y al óleo de principios del s. $X V$ a finales del s. XIX. Las técnicas analíticas empleadas les permitieron llegar a la conclusión de que en un proceso de limpieza normal no se produce la lixiviación de componentes de bajo peso molecular provenientes de la estructura pictórica, y que no existe el menor riesgo, siempre que el proceso sea realizado por restauradores experimentados. Dos años después, Sutherland (2000) presentó los resultados de otra investigación. En ella se demostraba que barnices preparados con resinas polares (como dammar o MS2A) pueden producir la lixiviación de ácidos grasos en estructuras pictóricas oleosas.

La cierta sensación de alarma que puede producir el trabajo de Sutherland con respecto a una operación aparentemente inocua se ve contrarrestada por las tranquilizadoras conclusiones del estudio de White y Roy acerca de un tipo de intervención considerada como mucho más perjudicial (por lo menos en potencia) para la conservación de la obra. ¿Qué conclusiones podemos sacar?

El artículo de White y Roy se planteó como una respuesta crítica a investigaciones anteriores, en especial a las presentadas en el congreso de Bruselas (véase más adelante) Cleaning, Retouching and Coatings (Mills y Smith, 1990). La principal objeción residía en que las pruebas no se realizaban en pinturas reales, envejecidas en condiciones normales, sino en muestras preparadas en laboratorio y sometidas a un envejecimiento acelerado. Además, también se criticaba que la forma de realizar los ensayos no se correspondía con las condiciones en las que un restaurador realiza un proceso de limpieza. Dicho de otro modo, que los resultados obtenidos, en los que se muestran fenómenos de lixiviación más o menos acusados, no reflejan lo que sucede realmente en una restauración realizada de forma correcta !

En este trabajo, no se va a analizar la forma más adecuada de realizar los ensayos de laboratorio para comprobar los efectos de los disolventes (un tema que merecería un estudio mucho más amplio y minucioso), aunque sí debe destacarse que esta cuestión es, justamente, uno de los puntos que generan las contradicciones y los debates. Es significativo que Sutherland (2000: 6I), al concluir su artículo, señale que los resultados obtenidos [...] en este estudio son deliberadamente exagerados, y no serán necesariamente aplicables de forma directa a una amplia gama de películas pictóricas encontradas en la práctica. Volvemos, pues, a la misma pregunta, iqué conclusiones podemos sacar? Para ello, para obtener algunas conclusiones, debemos analizar antes una serie de cuestiones previas y revisar también otras líneas de investigación.

\section{La estructura superficial como único campo operativo del proceso de limpieza}

Un axioma fundamental en la metodología del proceso de limpieza es que dicha operación se realiza en la estructura superficial, no en la estructura pictórica (Barros, 2000b). La estructura superficial incluye todos los depósitos presentes sobre el tejido pictórico, con independencia de su composición, función u origen (accidental o intencionado): barnices, colas, suciedad, repintes, estucos... Hay que subrayar que este concepto no implica una función determinada, ya que la estructura puede estar compuesta por materiales tan dispares como un barniz resinoso o excrementos de insectos. Tampoco implica que los materiales presentes sean originales o no (puede tratarse de una capa de barniz aplicada por el propio pintor). Ni siquiera implica una relación o conexión determinadas, física, estética o de cualquier otro tipo, con la estructura pictórica. Lo que únicamente define como tal a la estructura superficial es el hecho de ser el espacio donde opera el proceso de limpieza, es decir, donde se realiza la selección de materiales que pueden ser extraídos durante dicho proceso (Barros, 2000b: 396).

La limpieza se concibe así como una operación de selección y extracción de ciertos depósitos pertenecientes a la estructura superficial. Sólo aquí debe realizarse la intervención, sin afectar de ningún modo a los materiales no seleccionados para su extracción ni a la estructura pictórica. Esta última no puede ser modificada de ninguna forma, ni por adición (residuos) ni por sustracción (pérdida de pigmentos y/o del aglutinante). La intervención debe ser selectiva, progresiva y controlable. En definitiva, el concepto de estructura superficial define los límites físicos del proceso de limpieza en función de los propios límites de la estructura. Sólo dentro de este estrecho margen puede realizarse el proceso de selección de materiales extraíbles.

El concepto de estructura superficial responde a una necesidad nominal y metodológica, a una necesidad de ordenación, de designación y de establecimiento de límites aunque sólo sean conceptuales. Al hablar de límites puede plantearse una objeción. Se trata de cómo puede traducirse en el lenguaje operativo los 
imprecisos contornos de la estructura superficial. $\bigcirc$ dicho de otro modo, ¿dónde establecer la frontera física que el sistema de limpieza no debe traspasar de ningún modo? La interfase entre ambas estructuras puede, de hecho, no existir como tal límite definido. Así pues, ¿dónde debe establecerse tal línea divisoria?

Debe tenerse presente que en cada caso se establecen diferentes formas de enlace entre ambas estructuras (Barros, 2000b: 396-398). La forma en la que se configura la interfase debe analizarse de forma muy cuidadosa en cada nueva operación para establecer cuáles son las posibilidades de separación de cada una de las fases. El arco de posibilidades puede ir desde la fusión total entre ambas estructuras, hasta un límite claramente definido y que permita una separación fácil y neta. En la mayoría de los casos encontraremos situaciones intermedias. De todas formas, esta relación no puede establecerse a priori, sino sólo después de un estudio riguroso en el que se analicen todos los factores que intervienen en la configuración de una situación dada. Los más importantes, aunque no los únicos, son la composición de ambas estructuras y el grado de porosidad y cohesión de la estructura pictórica.

\section{Afinidades en la composición}

Uno de los factores que condicionan de forma más importante el proceso de limpieza es el hecho de que pueden existir evidentes afinidades entre la composición de las estructuras pictórica y superficial.

En la estructura superficial puede hallarse cualquier tipo de material, orgánico o inorgánico, natural o sintético, hidrófilo o lipófilo. Además de ello, los materiales pueden estar dispuestos en innumerables combinaciones, en cuanto al predominio de uno u otro o la localización dentro de la estructura. La variedad es tal, que hace imposible prever un modelo tipológico dado de estructura superficial. Las posibles combinaciones son prácticamente infinitas.

Además, los materiales que podemos hallar depositados tienen una inquietante semejanza con los que podemos encontrar en la pintura: aceites secantes, colas proteicas de origen animal, clara o yema de huevo, resinas naturales, pigmentos... Esta situación implica dos cuestiones de gran importancia. La primera es que la posibilidad de realizar una limpieza segura estará relacionada con la diferencia en la composición de ambas estructuras. Se tendrá una mayor garantía de éxito y de ausencia de interacciones con elementos no deseados cuanto mayor sea la diferencia. La segunda está en relación con el diseño de sistemas de limpieza más controlables y específicos. Además de los disolventes, se está empleando en la actualidad una amplia gama de herramientas (en un sentido amplio) que permiten un control mucho más preciso en la extracción de los materiales seleccionados. Sin embargo, muchos de estos nuevos sistemas también plantean problemas, en especial en cuanto a la posible permanencia de residuos no volátiles (Barros, 2000a).

\section{La estructura pictórica como cuerpo poroso}

Para comprender en qué medida los agentes de limpieza pueden poner en peligro la estabilidad material de la obra, hay que profundizar en la idea de la estructura pictórica como un cuerpo poroso. El estudio sobre los disolventes y su utilización, realizado por el IRPA (Masschelein-Kleiner, 198I), subrayaba la importancia del modelo de cuerpo poroso en el planteamiento de la limpieza. Lo relevante de este concepto es que ofrece una visión más completa de lo que ocurre en el interior de la pintura, concebida como una estructura tridimensional con una variada y compleja red de canales (fisuras, fracturas, poros). Éstos permiten el acceso de los disolventes al interior del cuerpo pictórico, hasta llegar incluso al propio soporte, y favorecen la retención de los mismos durante periodos de tiempo muy prolongados.

Los disolventes pueden desplazarse en profundidad, pero también paralelamente a la superficie, gracias a fracturas y poros que pueden permitir su acceso a zonas no deseadas. La estructura orográfica de cada pintura es fundamental para comprender cómo se produce el desplazamiento y retención de los disolventes (Southall, 1989: II-12; Michalski, 1990: 87; Zepeda Martínez, 1997).

La presión con la que penetra el líquido, que varía según la técnica de limpieza empleada, puede favorecer el desprendimiento de pequeñas partículas de película pictórica, tanto en la superficie como en el interior de los conductos por donde se desplaza el disolvente. Algunos factores que intervienen en la forma de desplazamiento del líquido por el interior de los conductos son la velocidad, la viscosidad y la geometría de propio conducto. Es importante controlar la cantidad de disolvente, la presión que se ejerce al limpiar y realizar previamente una completa y cuidadosa fijación de la pintura.

También debe tenerse en cuenta que los disolventes retenidos en la estructura pictórica presentarán posteriormente una difusión de retorno hacia la superficie, por lo que pueden seguir actuando sobre la película pictórica (Dauchot-Dehon, 1976; Ruhemann, 1982: 197 y 316; Michalski, 1990; Botticelli, 1992: 83).

\section{La lixiviación}

A partir de los años 30 se publicaron los primeros estudios que ofrecían un modelo de comportamiento de las películas oleosas en contacto con los disolventes. En 1936, G. L. Stout demostró que, en el proceso de disolución de estratos resinosos y oleosos, la hinchazón es un estado intermedio de la acción disolvente. 
En 1953, Graham presentó una serie de interesantes conclusiones, obtenidas al investigar la acción de disolventes sobre un estrato compuesto por sandáraca y aceite de lino. Estudió la velocidad de penetración de diferentes disolventes y comprobó la pérdida de moléculas de bajo peso molecular (lixiviación) durante la limpieza. Resultan de especial interés sus conclusiones acerca de la cuestión de la neutralización de disolventes ${ }^{2}$ : mezclas de esencia de trementina u otros hidrocarburos con alcohol etílico pueden hinchar una película de aceite de forma más intensa que el alcohol sólo. Una mezcla de alcohol etílico y esencia de trementina (60:40) es capaz de hinchar la película de aceite tres veces más que el alcohol puro. Sin embargo, este efecto sólo se consigue muy despacio, dependiendo de la duración de la exposición. Por ello la trementina puede prácticamente ser considerada un diluyente, pero nunca un medio de neutralización.

A partir de 1954 Nathan Stolow inició una importante investigación acerca de la acción de disolventes sobre películas de óleo, pigmentadas y no pigmentadas, en el Courtauld Institute of Art (Stolow, 1955) y en el laboratorio de la National Gallery of Canada (Stolow, |960-6I). A continuación se exponen, de forma muy breve, los resultados obtenidos en este estudio (Stolow, 1985).

\section{El modelo de Stolow}

Tras el secado de una pintura al óleo, permanecen, en el interior de la estructura del polímero, una serie de materiales solubles, triglicéridos no polimerizados y productos de degradación, que pueden ser extraídos por disolventes orgánicos. Cuando una película seca entra en contacto con un disolvente, se observa que éste penetra rápidamente, hinchando en primer lugar las partes externas, y cuando ha transcurrido el tiempo suficiente, la capa entera. Apenas el disolvente ha penetrado en la película, el material soluble puede difundirse hacia el exterior. A este fenómeno de extracción del material soluble se le denomina lixiviación. Cuando el disolvente comienza a evaporarse, la película comienza a deshincharse y al final tiene un volumen menor que el de partida (Stolow, 1985: 54-55).

La facilidad con la que los disolventes pueden extraer estos componentes solubles depende de diversos factores. Uno de los más importantes es la propia pintura. El grado de penetración de los disolventes es menor en las películas pigmentadas y, en especial, con pigmentos que faciliten el secado del aceite (como por ejemplo el blanco de plomo). Las propiedades de absorción de la luz de los pigmentos determinan también la acción fotoquímica sobre el aglutinante. La alta absorción de la luz UV por parte de algunos pigmentos puede acelerar la degradación de la estructura. Estos factores, entre otros, determinarán la resistencia de la estructura pictórica.

La aplicación de un disolvente produce cambios irreversibles a causa de la lixiviación: pérdida de peso, volumen y flexibilidad. En capas pigmentadas la con- tracción puede llegar al I 2\% del volumen, y la densidad puede incrementarse de forma significativa (Stolow, 1963).

La lixiviación de componentes solubles puede ocurrir en cualquier proceso de limpieza, dependiendo del tiempo de contacto del disolvente, tipo de disolvente, espesor de la película pictórica, edad y la propia composición de la pintura.

Las pérdidas máximas por lixiviación son del orden del $20-50 \%$ del aglutinante. En caso extremos puede llegar al $80 \%$, en películas muy degradadas y con escasa resistencia a los disolventes. Si se llega a una pérdida tan extrema se produce la desintegración de la estructura pictórica y la pérdida de pigmentos. Hay que insistir en que estos valores de lixiviación son los máximos que pueden alcanzarse, no los que se alcanzan en un proceso normal de limpieza realizado correctamente.

Aunque el grado de lixiviación sea bajo, el fenómeno no puede evitarse en ningún caso ${ }^{3}$. Lo único que puede hacer el restaurador es minimizar los daños al seguir unas normas muy elementales: reducir la acción mecánica, reducir el tiempo de contacto entre el disolvente y la superficie pictórica, emplear pequeñas cantidades de disolventes, permitir su adecuada evaporación y utilizar disolventes con bajo poder de hinchamiento y escasa difusión. Las aplicaciones repetidas de uno o varios disolventes en una misma área pueden provocar altas concentraciones en la estructura pictórica. Cualquier acción mecánica en esa zona, por leve que sea, podría causar graves daños.

Según Stolow, las películas pictóricas antiguas, de más de 300 años, presentan también, al entrar en contacto con disolventes, un proceso de lixiviación aunque el hinchamiento puede ser menor que en películas más recientes.

\section{Otros estudios sobre la lixiviación}

P.L. Jones presentó en 1965 las conclusiones obtenidas a partir del estudio de la lixiviación de películas de aceite de lino con alcohol isopropílico. Obtuvo resultados bastante parecidos a los de Stolow, con una solubilidad máxima del 30\%. La influencia de la iluminación con UV en la degradación de la pintura también fue confirmada, con un aumento en la solubilidad hasta un $70 \%$.

Tal como ya se ha señalado, en 1990 se presentaron los resultados de varios estudios en el Congreso de Bruselas (IIC). Erhardt y Tsang (1990) estudiaron el efecto de varios disolventes (hexano, tolueno, acetona y etanol) en capas de óleo con diferentes niveles de envejecimiento (5, 10 y 50 años) y con diferentes pigmentos (blanco de plomo, siena natural, bermellón y rojo azo). Los resultados obtenidos mostraron la lixiviación de pequeñas cantidades de ácidos grasos y productos de degradación. Se comprobó también que el porcentaje de componentes extraídos y 
los cambios en peso, espesor, color y brillo varían según el disolvente, pigmento, tiempo de contacto y edad de la muestra. Se produce una mayor lixiviación con acetona y tolueno, y en las muestras con siena. La presencia de blanco de plomo reduce la acción de los disolventes, incluso en películas recientes. Se confirmó también que una intensa lixiviación produce una disminución en el espesor y peso de la película pictórica. También produce un aumento de la luminosidad y una reducción del brillo. El examen de la superficie con SEM mostró la pérdida de pigmentos en algunas muestras.

Hedley, junto con otros investigadores (1990), estudió también las modificaciones que pueden producir los disolventes en películas de aceite de lino pigmentadas, y envejecidas durante 12 años. Las conclusiones son semejantes a los demás estudios, mostrando la importancia de los pigmentos y la mayor sensibilidad de las capas pigmentadas con siena tostada con respecto a las pigmentadas con blanco de plomo. Esto podría ser explicado por la mayor accesibilidad del disolvente y la mayor cantidad de aglutinante. Una intensa lixiviación, con la consiguiente pérdida de componentes de bajo peso molecular produce un claro aumento de la rigidez de la pintura.

Michalski (1990) presentó una interesante propuesta para la elaboración de un nuevo modelo físico del proceso de limpieza. Entre otros temas, planteó la relación existente entre la difusión y la disolución. Considera que un disolvente de baja difusión puede producir un mayor hinchamiento de la película pictórica si necesita mucho más tiempo para disolver el barniz. Esto significa que la relación entre la velocidad de difusión del disolvente y la velocidad con la que disuelve el barniz es la que determina la penetración en la pintura, no únicamente la difusión. Otra cuestión planteada por Michalski es la posible afinidad existente entre ciertos disolventes y pigmentos. Estas interacciones pueden provocar la ruptura del enlace pigmento-aglutinante y la pérdida de saturación del color. Sin embargo, se trata todavía de un tema poco estudiado.

Según Michalski, el proceso de alteración física de la estructura pictórica al entrar en contacto con disolventes pasa por dos etapas: I) se produce un hinchamiento del aglutinante en un grado muy variable (5$80 \%$ ), y en consecuencia su desplazamiento y compresión. Puede producirse la ruptura del enlace pigmento-aglutinante, en especial si el disolvente posee una afinidad con las partículas. El aglutinante es lixiviado; 2) El disolvente se evapora, secándose el aglutinante. Se produce una contracción de hasta un I5\% por la lixiviación y hasta un $80 \%$ por compresión plástica. Con respecto a las alteraciones ópticas señala que, por pequeñas que sean las pérdidas que puedan producirse en la superficie de una pintura, éstas originarán una modificación de la apariencia. La lixiviación puede producir la formación de microporos o la pérdida de cohesión de la superficie. Esto, a su vez, puede provocar pérdidas en el brillo y en la saturación del color y, en general, un efecto blanque- cino. Michalski subraya la importancia de la lixiviación, en especial en las pinturas no demasiado antiguas, en obras de los ss. XIX y XX.

En todas estas investigaciones se admite el peligro de la lixiviación pero también se muestra el grado real de incertidumbre que todavía impregna esta cuestión y las limitaciones en los conocimientos. Por lo general, se admite la validez del modelo de Stolow como un buen punto de partida para comprender qué sucede cuando el disolvente entra en contacto con la estructura pictórica. Sin embargo, se han señalado también numerosas limitaciones en los resultados de Stolow, en su mayor parte relacionadas con la forma de realizar las pruebas y el tipo de muestras utilizadas (Phenix, 1998).

No cabe duda que el riesgo de lixiviación es real. Lo que se cuestiona es la magnitud del fenómeno en un proceso de restauración realizado de forma correcta. En esta dirección apunta el trabajo de White y Roy, aunque sus resultados tampoco pueden considerarse como concluyentes. Se trata de un tema todavía abierto a nuevas investigaciones, y en especial cuando se trata de realizar pruebas en estructuras pictóricas con un aglutinante no exclusivamente oleoso.

\section{Los efectos de los disolventes orgánicos so- bre aglutinantes mixtos}

Los estudios sobre la lixiviación se han centrado en estructuras pictóricas compuestas exclusivamente por un aceite secante como aglutinante. Apenas se ha planteado la cuestión de cómo puede afectar este fenómeno a mezclas más complejas como emulsiones o pinturas oleosas con diferentes aditivos como ceras o resinas.

Erhardt y Tsang (1990: 97) han señalado que ciertos aditivos presentes en una pintura al óleo, como cera o resina, pueden ser extraídos fácilmente por la acción de los disolventes. Se ha puesto de manifiesto la necesidad de un completo estudio, en especial en el complejo caso de las pinturas del s. XIX, donde es frecuente la presencia de muy diversas mezclas $y$ combinaciones de aglutinantes.

Jaap J. Boon y Jos van Och (1996) han estudiado el problema de la lixiviación en una pintura al óleo con una importante presencia de cera de abejas. La investigación ha mostrado que los disolventes producen la lixiviación de ácidos grasos y su arrastre hacia la capa de preparación.

Sin embargo, no se ha estudiado el proceso de limpieza en estructuras con otro tipo de aglutinantes mixtos, como los óleo-proteicos, muy frecuentes en los ss. XV-XVI, y recuperados en el s. XIX. Sí se ha realizado una aproximación al problema en el caso de los aglutinantes exclusivamente proteicos, aunque también en este caso los resultados son todavía preliminares. 
Los efectos de los disolventes orgánicos sobre materiales proteicos

Una investigación realizada por Adam Karpowicz (198I) ha mostrado que la acción de los disolventes orgánicos sobre los materiales proteicos no es tan inocua como podría parecer en un principio. Karpowicz considera que las pinturas con estos aglutinantes, con una estructura relativamente estable creada durante el envejecimiento, pueden ser debilitadas o por lo menos sufrir cambios impredecibles durante los tratamientos de limpieza. El efecto más intenso podría ser ejercido por algunos alcoholes, que podrían provocar un cambio importante en las propiedades químicas y físicas de las proteínas y hacerlas vulnerables a la acción de diversos agentes químicos y atmosféricos.

En otro estudio, realizado por Nharayan Khandemar, Alan Phenix y Julia Sharp (1994), se muestra que los disolventes también pueden producir en este tipo de aglutinantes la extracción de compuestos de bajo peso molecular, como ciertos ácidos grasos. Aunque el temple de huevo es considerado como un aglutinante proteico (la desnaturalización de las proteínas forma la película seca), contiene una gran cantidad de lípidos (64,7 \% en la película seca). En las películas jóvenes se produce una rápida extracción de lípidos en las pruebas realizadas bajo inmersión y con hisopo, empleando disolventes orgánicos. Esta extracción es acompañada, en algunos casos, por una disgregación física de la pintura. Los factores más importantes que determinan el grado de lixiviación son el tipo de disolvente utilizado, tiempo de exposición y la edad y pigmentación del estrato pictórico. La capacidad de los disolventes para lixiviar material soluble disminuye cuando las películas son sometidas a un envejecimiento acelerado con luz 4 .

Estas investigaciones son todavía preliminares. Posteriores estudios deben aportar una información más detallada y exacta sobre los efectos de los disolventes orgánicos en las capas proteicas. Aunque en condiciones normales de limpieza, estos aglutinantes parecen ser bastante resistentes a los disolventes, deben tomarse todas las precauciones necesarias para limitar su acción.

\section{El blanqueamiento de la película pictórica}

Tras una operación de limpieza con disolventes o reactivos se puede observar, en algunos casos, la aparición de unas manchas blanquecinas. Éstas suelen mostrarse como una señal inequívoca de daños en el tejido pictórico. Sin embargo, esta cuestión también plantea aspectos que merecen un estudio más riguroso.

Según Erhardt y Bischoff (1993: | 43), las causas de este fenómeno pueden ser las siguientes: ( I) extracción de material soluble de la pintura, (2) ruptura del enlace pigmento-aglutinante, (3) excesiva hinchazón de la estructura del aglutinante y (4) cambios en la textura superficial de la película pictórica. Cada una de estas acciones puede producir la aparición de huecos en el tejido pictórico al evaporarse el disolvente. El resultado final es que el color aparece más luminoso y blanquecino. Este efecto puede ser anulado rellenando los huecos de la superficie con un material no volátil como un barniz. El éxito de una intervención de limpieza debe juzgarse por el grado en el que estos efectos indeseables son evitados, y el grado en el cual la pintura se asemeja a una pintura limpia pero no limpiada. Normalmente, para comprobar si la operación ha tenido poco efecto sobre la estructura pictórica deben observarse los siguiente factores: (I) una superficie lisa o brillante, (2) ausencia de pérdida de pigmentos y (3) ausencia de blanqueamiento.

Sin embargo, el blanqueamiento causado por una acción excesiva sobre el aglutinante no debe confundirse con el efecto causado por la permanencia de restos de barniz en la superficie. Si el desbarnizado no es completo y se realiza con disolventes de evaporación muy rápida, los restos de barniz pueden producir un efecto de mancha blancuzca sobre la superficie de la pintura. La observación de la acción de la acetona sobre algunos barnices, preparados a partir de las resinas dammar y almáciga, muestra que la rápida evaporación del disolvente produce la ruptura de la capa de barniz que no ha sido extraída (Burnstock y White, 1990: I 13). La progresiva pérdida de contacto entre la pintura y los fragmentos de resina causa un incremento en la dispersión de la luz. Este efecto ha sido señalado por Ruhemann (1982: I 86 y 197) y otros autores (Bergeon et al., 1981; Burnstock, Caldwell y Odlyha, 1993: 236).

El consumo de calor durante la evaporación de un líquido hace que en ciertos casos se llegue a la temperatura de condensación del agua. Esto provoca la condensación de la humedad contenida en el aire cercano a la superficie de evaporación. Este fenómeno también puede provocar la aparición de manchas blanquecinas si la pintura contiene sustancias sensibles al agua, como algunas resinas terpénicas, o si se han empleado, en la limpieza, disolventes de rápida evaporación como el isooctano o el éter (Masschelein-Kleiner, 1981: 29).

En cualquier caso la presencia de un efecto de blanqueamiento debe comprobarse siempre. Si aparece de forma reiterada puede ser una clara señal de que se han producido daños en el aglutinante, en especial si éste presenta aditivos fácilmente solubles.

\section{La retención de residuos: tensoactivos}

La retención de residuos en una estructura pictórica ha sido analizada con relación a muchos de los materiales utilizados en las limpiezas. Los estudios del IRPA, en los años 70-80, demostraron la importancia de este problema cuando se emplean disolventes orgánicos y reactivos. En los últimos años, otros estu- 
dios se están centrando en la retención de residuos de materiales no volátiles, en especial tensoactivos y geles. Las aportaciones del restaurador y bioquímico Richard Wolbers han dado lugar a numerosas investigaciones sobre los posibles problemas que podrían provocar ciertos componentes, principalmente en el caso de los detergentes o de los llamados jabones de resina (Barros, 1998).

\section{Residuos y cambios visuales}

Las propiedades ópticas y la apariencia de una pintura son, en general, debidas a las características de superficie del tejido pictórico. Esto indica que la permanencia de residuos puede tener un efecto significativo en la apariencia que presente la pintura.

Wolbers defiende la utilización de los jabones de resina ${ }^{5}$ porque, entre otras ventajas, no dejan una superficie blanquecina, tal como ocurre en muchas ocasiones con los disolventes orgánicos. Burnstock y White (1990: 115) consideran que la permanencia de restos de los jabones y, en especial, del Tritón X100 (un detergente) podría ser la causa del aparente brillo saludable que presenta la superficie tras la limpieza. Erhardt y Bischoff (1994: 10- I I y 19) han sugerido también que el aspecto saturado de las películas tratadas con estos jabones puede ser debido a la saturación del estrato pictórico con residuos no volátiles del propio jabón.

El aspecto blanquecino de una película pictórica tras una limpieza con disolventes puede ser un indicio claro del uso incorrecto de los mismos, pero la ausencia de esta alteración visual no indica necesariamente que no se haya producido algún deterioro. Esto es aplicable a los jabones de resina, que producen efectos ópticos opuestos a los de los disolventes. Las propiedades ópticas dependen de las características de la superficie. Los valores exactos de color, luminosidad y brillo son muy sensibles a la pérdida o incorporación de material y a pequeños cambios en la textura (Erhardt y Bischoff, 1994: I I).

\section{Residuos y alteraciones químicas}

Otro problema que plantean los residuos de estos jabones es que puedan permanecer activos. Al evaporarse el agua la concentración de los componentes no volátiles se incrementa y algunos de ellos presentan propiedades que podrían resultar peligrosas para la conservación de la estructura pictórica. Estas mezclas concentradas podrían ser más activas que la mezcla inicial, más diluida (Erhardt y Bischoff, 1994: 19).

En un estudio efectuado por Burnstock y White (1990: 113-114) se encontraron restos de los jabones en las áreas donde fueron utilizados y después aclarados con White Spirit o saliva. Se detectaron restos de los compuestos de los ácidos deoxicólico y abiético y trazas de algunos compuestos pertenecientes al Tritón X-100, un éter, siendo por lo tanto muy susceptible de formar peróxidos orgánicos que, a su vez, pueden iniciar la formación de radicales libres (White, 1993). Los radicales libres pueden acelerar la autooxidación del óleo o de componentes resinosos.

La trietanolamina (TEA) es una amina orgánica que se combina con los ácidos para formar el jabón. La TEA es un líquido poco volátil y extremadamente higroscópico, por lo que puede retener agua y permanecer parcialmente hidratado. Además, puede oscurecer por exposición al aire y a la luz (Erhardt y Bischoff, 1994: 4). Algunos experimentos han mostrado que una mezcla de TEA y ácido abiético absorbe alrededor de un 10\% de su peso en agua de la atmósfera para formar un semi-sólido a $20^{\circ} \mathrm{C}$ y $50 \%$ HR. La TEA absorbe casi un 20\% de su peso en agua bajo las mismas condiciones. Erhardt y Bischoff consideran que, aunque no se conocen los efectos a largo plazo de estos residuos sobre la pintura, puede pensarse que podrían producir, entre otras alteraciones, una hidrólisis parcial del aglutinante oleoso acelerada por la presencia de agua (Erhardt y Bischoff, 1994: 18-19). En el mismo estudio se ha comprobado, además, que en algunas de las muestras la TEA residual no ha permanecido en la superficie, sino que ha sido absorbida: parece haber plastificado y ablandado el aglutinante oleoso, produciendo el arrugamiento del tejido pictórico (Erhardt y Bischoff, 1994: 14 y 19).

Como podemos comprobar, muchas reflexiones apuntan hacia la compleja cuestión de los efectos que puedan tener en el futuro los residuos de los tensoactivos. Sin embargo, no sólo los tensoactivos plantean este tipo de problema.

\section{La retención de residuos: los geles de Carbopol}

En la última década se viene comprobando la utilización cada vez mayor de geles y pastas para obtener un mayor control de disolventes, reactivos o enzimas. De esta forma se pretende restringir su acción a los materiales de la estructura superficial seleccionados para su eliminación. Sin embargo, también se trata de compuestos no volátiles, lo que plantea nuevamente el problema de la extracción de los residuos. Un ejemplo de esta situación es el empleo de los geles preparados con Carbopol.

Las resinas Carbopol son polímeros del ácido acrilico, ácidos en medio acuoso ( $\mathrm{pH}$ 2,8-3,2). Su neutralización con aminas es indispensable para su utilización como espesantes inertes. Suele recomendarse el Ethomeen $\mathrm{Cl} 2$ y el Ethomeen C25 para la formación del gel con el Carbopol, el Cl 2 para geles con hidrocarburos aromáticos y el C25 para geles con disolventes polares, incluyendo el agua. Estos reactivos son muy básicos y forman una sal con el Carbopol por la presencia del grupo amina. La información aportada por los fabricantes indica que poseen, aproximadamente, un 3\% de aditivos, lo que incluye aminas primarias y secundarias (Burnstock y Kieslich, 1996: 253). 
Un aclarado efectivo debe permitir la eliminación de todos los compuestos no volátiles del gel, lo que incluye la masa de Carbopol y Ethomeen. Wolbers sugiere Shellsol A para la eliminación de un gel con disolventes de baja polaridad o la misma mezcla de disolventes utilizados en el gel. Otros autores recomiendan sólo hidrocarburos aromáticos o hidrocarburos tras un primer aclarado con saliva o agua. Aviva Burnstock y Tanya Kieslich (1996) han estudiado estos sistemas de aclarado. La conclusión a la que han llegado es que los diversos procedimientos eliminan selectivamente los componentes del Ethomeen. Por ejemplo, las aminas primarias se eliminan con el aclarado con saliva seguido de la mezcla de hidrocarburos. Sin embargo, y a pesar de que se consigue eliminar la práctica totalidad del gel, permanecen siempre residuos de las aminas secundarias.

Este estudio preliminar ha puesto de manifiesto la necesidad de una investigación más profunda en la que se plantee también la posibilidad de la retención de residuos del Carbopol y los efectos a largo plazo de los depósitos residuales de aminas secundarias y otros componentes del Ethomeen.

\section{Conclusiones}

Las conclusiones que pueden obtenerse con respecto al problema de si un proceso de limpieza es o no seguro para la adecuada conservación de la estructura pictórica no son tranquilizadoras ni cómodas. Buena parte de las investigaciones comentadas nos muestran el elevado nivel de incertidumbre que domina este tipo de intervención. Tienen, además, la virtud de mostrarnos de forma clara lo que puede suceder si no se sigue una óptima metodología.

Si nos centramos únicamente en la cuestión de la lixiviación, podemos comprobar que es muy difícil predecir el grado de riesgo ante el que nos enfrentamos. En todo caso, sí se pueden tener en cuenta una serie de factores. Por una parte, los relacionados con la propia estructura pictórica: ( I) tipo de aglutinante (aceite secante, proteico...), (2) presencia de aditivos solubles (ceras, resinas...), (3) tipo de pigmento (influencia en la estabilidad del aglutinante), (4) presencia de pigmentos solubles, (5) proporción pigmento/aglutinante, (6) grado de cohesión, (7) espesor y (8) topografía y estructura interna (cuarteado, porosidad, textura...). Por otra parte, los relacionados con el proceso de limpieza: ( I ) interacción entre el disolvente y el aglutinante, (2) interacción entre el disolvente y los pigmentos, (3) velocidad de difusión del disolvente, (4) grado de retención del disolvente, (5) cantidad de disolvente utilizado, (6) acción mecánica (grado de abrasión) y (7) tiempo de contacto entre el disolvente y la superficie pictórica.

Este es un ejemplo de que el problema no puede reducirse a unos pocos factores, sino que implica un abanico muy amplio, lo que exige una metodología de trabajo extremadamente rigurosa, que permita al restaurador preparar la estrategia óptima.

Por otro lado, también resulta evidente que todavía desconocemos demasiados aspectos relacionados con el comportamiento físico-químico y los cambios producidos durante el envejecimiento de la estructura pictórica. El propio proceso de limpieza plantea numerosos interrogantes, lo que hace imprescindible un estudio más amplio del tipo de ensayos que deben realizarse (Phenix, 1998), del mecanismo de actuación de los sistemas de limpieza, del efecto en una más amplia gama de aglutinantes (no sólo óleo) y del problema de los residuos.

\section{Agradecimientos}

Este artículo está basado parcialmente en la tesis doctoral Objetivos y límites en la limpieza de estructuras pictóricas, realizada bajo la dirección del Dr. Daniel Benito Goerlich y la Dra. Cármen Pérez García, a los que quiero manifestar mi agradecimiento por su inestimable ayuda. Esta tesis fue presentada y defendida el II de febrero de 2000 (Departamento de Conservación y Restauración de Bienes Culturales, Universidad Politécnica de Valencia).

\section{Bibliografía}

BARROS, JOSÉ MANUEL (1998): Las técnicas de Richard Wolbers: un examen crítico. En Actas del XII Congreso de Conservación y Restauración de Bienes Culturales. Alicante. pp. 56I-574.

BARROS, J. M. (2000): Fundamentos metodológicos en la limpieza de estructuras pictóricas: una visión objetiva. $\mathrm{PH}$ : Boletín del IAPH, n 30, pp. 75-84.

BARROS, J. M. (2000a): Nuevos sistemas de limpieza de pinturas. Soluciones y problemas. Restauración \& Rehabilitación, $n^{\circ}$ 44, pp. 70-75.

BARROS, J. M. (2000b): El proceso de limpieza de obras pictóricas: un planteamiento estructuralista. En Actas del XIII Congreso de Conservación y Restauración de Bienes Culturales. Lleida. pp. 395-405.

BERGEON, S. et al. (198I): Le blanchiment: un cas precis d'étude. En 6th Triennial Meeting. Ottawa. 81/20/3.
BOON, JAAP J. y VAN OCH, JOS (1996): A mass spectrometric study of the effect of varnish removal from a 19th century solvent-sensitive wax oil painting. En I I th Triennial Meeting. Edinburgh, Scotland, I-6 september 1996. London: James \& James (Science Publishers) Ltd., ICOM Committee for Conservation. vol. I, pp. 197-205

BOTTICELLI, G. (1992): Metodologia di restauro delle pitture murali. Firenze: Centro Di.

BURNSTOCK, AVIVA, CALDWELL, MELANIE y ODLYHA, MARIANNE (1993): A technical examination of surface deterioration of Stanley Spencer's paintings at Sandham Memorial Chapel. En 10th Triennial Meeting. Washington, D.C., 22-27 August 1993. ICOM Committee for Conservation. vol. I, pp. 231-238.

BURNSTOCK, A. y KIESLICH, TANYA (1996): A study of the clearance of solvent gels used for varnish removal from paintings. En I / th Triennial Meeting. Edinburgh, Scotland, I-6 september 
1996. London: James \& James (Science Publishers) Ltd., ICOM Committee for Conservation. vol. I, pp. 253-262.

BURNSTOCK, A. y WHITE, R. (1990): The effects of selected so I vents and soaps on a simulated canvas painting. En Cleaning, Retouching and Coatings. London: ||C, pp. I | | - | | 8 .

DAUCHOT-DEHON, MICHÈLE (1976): Etude de la retention des solvants par traceur dans les peintures. En Applicazione dei metodi nucleari nel campo delle opere d'arte. Roma Accademia Nazionale dei Lincei. pp. 567-576.

ERHARDT, DAVID y BISCHOFF, JUDITH J. (1993): Resin soaps and solvents in the cleaning of paintings: similarities and differences. En 10th Triennial Meeting. Washington, D.C., 22-27 August 1993. ICOM Committee for Conservation. vol. I, pp. |4|- |46.

ERHARDT, D. y BISCHOFF. J. J. (1994): The role of various components of resin soaps, bile acid soaps and gels, and their effects on oil paint films. Studies in Conservation, vol. $39 n^{\circ}$ I, pp. 3-27.

ERHARDT, D. y TSANG, JIA-SUN (1990): The extractable components of oil paint films. En Cleaning, Retouching and Coatings. London: IIC, pp. 93-97

GRAHAM, IAN (1953): The effect of solvents on linoxyn films. Journal of the Oil and Colour Chemists Association 36, 1953, pp. 500-506.

HEDLEY, G. et al. (1990): A study of the mechanical and surface properties of oil paint films treated with organic solvents and water. En Cleaning, Retouching and Coatings. London: IIC, pp. 98-I05.

JONES, P.L. (1965): The leaching of linseed oil films in isopropyl alcohol. Studies in Conservation, vol. 10 n³, pp. I19-129.

KHANDEMAR, NARAYAN, PHENIX, Alan y SHARP, Julia (1994): Pilot study into the effects of solvents on artificially aged egg tempera films. The Conservator, $n^{\circ}$ I8, pp. 62-72.

KARPOWICZ, ADAM (198I): Ageing and deterioration of proteinaceous media. Studies in Conservation 26, n 4, pp. I53- 160.

MASSCHELEIN-KLEINER, L. (198I): Les solvants. Cours de conservation 2. Bruxelles: IRPA.

MICHALSKI, STEFAN (1990): A physical model of the cleaning of oil paint. En Cleaning, Retouching and Coatings. London: IIC, pp. 85-92.

MILLS, J. S. y SMITH, P. (eds.) (1990): Cleaning, Retouching and Coatings: Technology and Practice for Easel Paintings and Polychrome Sculpture. London: IIC.
MILLS, J. y WHITE. R. (1987): The Organic Chemistry of Museum Objects. London: Butterworths.

PHENIX, ALAN (1998): Solvent-induced swelling of paint films: some preliminary results. WAAC Newsletter $20 n^{\circ} 3$.

RUHEMANN, H. (1982): The Cleaning of Paintings. New York:

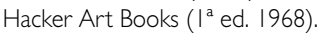

SOUTHALL, A. (1989): Wolbers' Corner. Conservation News, $n^{\circ}$ 39, pp. II-12.

STOLOW, NATHAN (1955): Some Investigations of the Action of Solvents on Drying Oil Films. Ph.D. thesis. London: University of London. Reeditado en dos partes en J. Oil and Colour Chemists' Assoc. 40, 1957. l: pp. 337-402. II: pp. 488-499.

STOLOW, N. (1960-61): Unpublished researches on solvent action in the Conservation and Scientific Research Division of the National Gallery of Canada. Ottawa.

STOLOW, N. (1963): Aplication of science to cleaning methods: solvent action studies on pigmented and unpigmented linseed oil films. En THOMSON, G. (ed.). Recent Advances in Conservation. London: Butterworths.

STOLOW, N . (1985): Solvent action: some fundamental researches into the picture-cleaning problem. En FELLER, R.L., STOLOW, N. y JONES, E. H. On picture varnishes and their solvents. Washington: National Gallery of Art, pp. 45- I 16. (Org. 1959; ed. revisada 197I).

STOUT, G. L. (1936): A preliminary test of varnish solubility. Technical Studies in the Field of the Fine Arts 4, n³, pp. |46-161.

SUTHERLAND, KEN (2000): The extraction of soluble components from an oil paint film by a varnish solution. Studies in Conservation 45, pp. 54-62.

WHITE, R. (1993): Estudios científicos relacionados con la limpieza de obras de arte con geles. En Memoria de actividades $1992-$ 1993. Valencia: ACRACV.

WHITE, RAYMOND y ROY, ASHOK (1998): GC-MS and SEM studies on the effects of solvent cleaning on old master paintings from the National Gallery, London. Studies in Conservation 43, pp. 159-176.

ZEPEDA MARTÍNEZ, Ma GUADALUPE (1997): Uso de geles en la limpieza gradual de pinturas sobre lienzo. México, D.F.: Instituto Nacional de Antropología e Historia.

\section{Notas}

I. Este tipo de críticas fueron también las empleadas por el restaurador Helmut Ruhemann al impugnar las investigaciones de Stolow (Phenix, 1998; Barros, 2000: 81).

2. La técnica usual de limpieza con disolventes supone el uso de un disolvente activo y otro que se utiliza para el aclarado. Este aclarado tiene el aspecto negativo de introducir una nueva cantidad de disolvente, que también debe evaporarse. El aspecto positivo es que de esta forma, la mezcla se hace menos activa.

3. Se trata de un proceso totalmente irreversible. Los intentos de mezclar plastificantes o suavizantes (como aceites) con los disolventes, para regenerar la película lixiviada, son totalmente inútiles (Stolow, 1963).
4. Debe señalarse que Mills y White (1987) también han indicado que los disolventes pueden producir la extracción parcial de lípidos en antiguos estratos de pintura al temple.

5. El término jabones de resina suele referirse genéricamente a la serie de jabones preparados por Wolbers a partir de ácidos terpénicos (ácido abiético) o ácidos bílicos (ácido deoxicólico). Las fórmulas más usuales incluyen: agua, el ácido orgánico, una amina orgánica (trietanolamina), un espesante y aditivos como el Tritón X-I00 (un detergente) o el alcohol bencílico (un disolvente). 